since 1997. The target group of the TRC consists of doctors and health care professionals. The primary aim of the TRC is to provide a standard training and enhance the quality of service. The certificates, which is valid for five years, have been given to the professionals who have succeeded in the theoretical and practical exams.

Methods: The authors have conducted an epidemiologicdescriptive method in this study. In the scope of the study, 90 professionals were called for four different courses, and 82 of them attended the theoretical and practical training. The gathered data were evaluated by making frequency distributions.

Results: In all, 63.4\% ( $\mathrm{f}=52)$ of the participants consisted of women and $73.1 \%(f=60)$ are high school graduates. The practice skills of the participants were classified under the eight titles. Respectively mean score of them; the Mean Score (MS) of providing advanced airway (intubation) is 18.9/20; MS of establishing vascular access and intraosseous attempt is 9.9/10; MS of stabilizing the neck and vertebra is 19.8/20; MS of the centric extraction device (CED) is 9.9/10; MS of the traction splint is 9.7/10; MS of the vacuum stretcher is 9.3/10; normal stretcher is $9.5 / 10$, and combination stretcher is 9.8/10. The general mean score of the theoretical exam is 83.6, practicing mean score is 96.9 , and the general success average is 90.2 .

Conclusion: The TRC scores of the CEMS personnel have been found to be quite high.

Prehosp Disaster Med 2017;32(Suppl. 1):s99-s100

doi:10.1017/S1049023X17002564

\section{No Cost Solutions to Performance-Based Disaster Medical}

\section{Education}

Lancer A. Scott ${ }^{1}$, Kemp Anderson ${ }^{2}$

1. Div Of Em, Medical University of South Carolina, Charleston/ SC/United States of America

2. Medical University of South Carolina, Charleston/SC/United States of America

Study/Objective: High-quality clinical disaster medicine requires medical teams working in chaotic environments. Many disaster education providers fail to adequately assess disaster performance during training, often concerned with the cost and complexity of such assessments. We created a competencybased, 5-hour Emergency Preparedness Training (EPT) curriculum with low-fidelity training tools and easy to reproduce skill assessments to improve trainee knowledge, confidence, and disaster medicine skills.

Background: High-quality clinical disaster medicine requires medical teams working in chaotic environments. Many disaster education providers fail to adequately assess disaster performance during training, often concerned with the cost and complexity of such assessments.

Methods: Diverse groups of medical university students, health care professionals, and community members were enrolled between 2011 and 2014. The course consisted of an online questionnaire, didactic lectures, small group exercises, and two live, multi-patient, mass-casualty incident (MCI) scenarios.
Results: All 708 participants completed the course. They were trained over three years, including $49.9 \%$ physicians, $31.9 \%$ medical students, $7.2 \%$ nurses, and $11 \%$ various other health care professionals. All $100 \%$ of the participants completed the pretest and $71.9 \%$ completed the posttest, with average correct answers increasing from 39\% to $60 \%$. Trainees met $73 \%$ and $96 \%$ of performance objectives during small group exercises and 68.5\% and $61.1 \%$ during the two MCI scenarios. Both overall knowledge and confidence with clinical disasters improved from 33/100 to $74 / 100$ (overall knowledge), and $33 / 100$ to $77 / 100$ (overall confidence). $91.5 \%$ of trainees highly recommended the course. Average cost of training was less than US $\$ 100$ per course.

Conclusion: Simple EPT design elements can improve trainee knowledge, confidence, and disaster medicine skills at a very low cost. This unique EPT curriculum may help educators with limited resources implement performance-based medical team training effectively and efficiently.

Prehosp Disaster Med 2017;32(Suppl. 1):s100

doi:10.1017/S1049023X17002576

\section{Legal Accountability of International Emergency Medical Teams In Disasters \\ Patrycja Szepczynska, Johan Von Scbreeb}

Department Of Public Health Sciences, Karolinska Institutet Centre for Research on Health Care in Disasters, Stockholm/Sweden

Study/Objective: This research maps and explores existing legal systems that, within a disaster context, may be applied to hold International Emergency Medical Teams accountable for medical errors.

Background: International Emergency Medical Teams (IEMT) provide clinical care to populations affected by disasters. However, while well intended, their work may result in harm to a patient. To date the medical legal accountability of IEMTs has not been systematically assessed.

Methods: This study is a narrative literature review. An initial search in Google and other search engines was performed. Legal documents, guidelines and grey literature referring to legal accountability of IEMTs were selected. Results were organized in two categories: international legal system and national legal system.

Results: IEMTs are deployed by different relief agencies, including governmental or non-governmental organizations, which are subjects to separate legal systems, for instance, of their own countries or international laws. No international laws related to disasters provide for mechanisms of IEMT's legal accountability. However, there are non-disaster international legal systems applicable to certain types of relief agencies deploying IEMTs - for instance, regional human rights systems. No database provides for a list of national legislations relevant to IEMTs, nevertheless the research confirmed that national disaster or nondisaster laws could be applied. There is no record of any legal case against an IETM decided in favor of a patient.

Conclusion:

- There is no specific legal system designed for enforcing legal accountability of IEMTs. 
- Due to differences in legal status of IEMTs' providers and national laws, similar events may end up with a different legal outcome.

- Patients may be devoid of mechanisms to seek redress, due to lack of relevant legal system applicable to their case, or when the applicable legal system provides for exemptions from accountability by means of judicial immunities or Good Samaritan laws.

- Ensuring medical liability insurance for IEMTs should be considered.

Prehosp Disaster Med 2017;32(Suppl. 1):s100-s101

doi:10.1017/S1049023X17002588

Developing AUSMAT's Rehabilitation Capacity: Applying the Technical Standards to Practice

Erica Bleakley

National Critical Care And Trauma Response Centre, Royal Darwin Hospital, Darwin/NT/Australia

Study/Objective: The objective of this case study is to describe the process undertaken by the Australian Medical Assistance Team (AUSMAT) in developing its rehabilitation capability, and applying the World Health Organization Classification and Minimum Standards for Emergency Medical Teams to practice.

Background: The Australian Medical Assistance Team is Australia's health emergency and medical response facility. In October 2016, AUSMAT achieved classification as a World Health Organization Type-2 Emergency Medical Team and field hospital. This achievement was the culmination of many months of preparation, by people from a wide range of areas of expertise, and served as the impetus for AUSMAT to address the need for rehabilitation within its broader capabilities.

Methods: Development of AUSMAT's rehabilitation capability required a process of rationalizing the minimum standards against AUSMAT's core business.

Results: AUSMAT's rehabilitation capability is closely integrated with its core clinical activities. AUSMAT rehabilitation professionals will work within a transdisciplinary model of practice between Occupational Therapy and Physiotherapy. The primary goals of the rehabilitation capability is to reduce secondary injury, achieve optimal outcomes post injury, improve patient flow through the field hospital and identify appropriate transfer and referral pathways. In accordance with typical AUSMAT tasking, the focus of the rehabilitation capability is on the acute phases post injury. Rehabilitation currently remains a largely untested capability for AUSMAT, however through the process of addressing the standards for rehabilitation in a Type-2 EMT, AUSMAT is now in a position to deploy rehabilitation professionals alongside medical, nursing, logistical and other team members.

Conclusion: AUSMAT has developed its rehabilitation capability, and has demonstrated to the WHO, core and technical standards for a Type-2 EMT and field hospital. As such, AUSMAT represents an example of operationalization of the minimum standards for clinical practice.

Prehosp Disaster Med 2017;32(Suppl. 1):s101

doi:10.1017/S1049023X1700259X
Perceptions and Reflections of Emergency Medicine Graduates, Regarding the Toronto Addis Ababa Academic Collaboration in Emergency Medicine (TAAAC-EM)

Curriculum: A Qualitative Evaluation Study

Elayna Fremes ${ }^{1}$, Cheryl Hunchak ${ }^{2}$, Sofia Kebede ${ }^{3}$, Nazanin Meshkat ${ }^{4}$

1. Toronto Addis Ababa Academic Collaboration in Emergency Medicine, Toronto/ON/Canada

2. Schwartz-reisman Emergency Medicine Centre, Mount Sinai Hospital, Toronto/ON/Canada

3. Black Lion Hospital, Addis Ababa/Ethiopia

4. University Health Network, Toronto/ON/Canada

Study/Objective: This study is a qualitative curriculum evaluation of the Toronto Addis Ababa Academic Collaboration in Emergency Medicine postgraduate training program, in Emergency Medicine at Addis Ababa University (AAU), Ethiopia.

Background: In 2010, the first-ever Emergency Medicine postgraduate training program in Ethiopia was launched at Addis Ababa University. Toronto Addis Ababa Academic Collaboration in Emergency Medicine (TAAAC-EM) designed and implemented a curriculum to support the Addis Ababa University (AAU) EM program. To date, three cohorts of EM specialists $(n=15)$ have graduated from the three-year program. After six years of implementation, we undertook a qualitative evaluation of the TAAAC-EM curriculum.

Methods: Data collection took place in 2016 in Ethiopia via in-person graduate interviews $(n=12)$. Participants were interviewed by a trained research assistant, who used a semistructured interview guide. Standard interview, transcription, and analysis protocols were utilized. Qualitative software (QSR-NVIVO 9) was used for thematic grouping and analysis. Results: Graduates of AAU's EM residency training program reported very positive experiences with the curriculum overall. All graduates recognized the importance of TAAAC-EM's emphasis on bedside teaching to their learning, a unique component of the TAAAC-EM model compared to the baseline teaching milieu at AAU. In addition, several themes emerged when graduates were asked about areas of program improvement, including: (1) shifting didactic clinical epidemiology teaching to the senior residency years (PGY2-3), to coincide with completion of a required residency research project; (2) increasing simulation and procedural teaching sessions; and (3) adding formal certification courses such as ATLS and ACLS.

Conclusion: Interviewing graduates of AAU's EM residency training program proved to be an important avenue for determining areas of curriculum improvement for future trainees. It also provided critical input to TAAAC-EM strategic planning discussions, as the partnership considers expanding its scope beyond Addis Ababa.

Prehosp Disaster Med 2017;32(Suppl. 1):s101

doi:10.1017/S1049023X17002606

Hospital Workload for Weapon-Wounded Females Treated by the International Committee of the Red Cross - More Work Needed than for Males

Peter Andersson ${ }^{1}$, Måns Mubrbeck ${ }^{2}$, Harald Veen ${ }^{3}$, Zaher Osman ${ }^{3}$, Johan Von Schreeb ${ }^{4}$ 